\title{
MAXWELLIAN DISTRIBUTIONS FOR STELLAR ROTATIONS
}

\author{
ARMIN J. DEUTSCH \\ Hale Observatories, \\ Carnegie Institution of Washington and California Institute of Technology, Pasadena, Calif., U.S.A.
}

\begin{abstract}
For stars in the main-sequence band, the distributions of 'line-widths' $V \sin i$ are rediscussed, with the inclusion of recent measurements by Walker and Hodge for B stars, and by Preston for Ap stars. The Ap stars are counted with normal stars of the same color-class. Over the whole range from B2 to A2, the observed incidence of sharp-line stars far exceeds the incidence predicted on the hypothesis that the true rotational velocities have a Maxwellian distribution. The 'extra' slow rotators are probably close binary remnants, in the sense of Van den Heuvel. However, it remains unclear how to distinguish individual members of this group of stars.
\end{abstract}

\section{Introduction}

For the Greenbelt Symposium in 1965, an attempt was made to find whether the line-width measurements of Slettebak and Howard (1955) are conformable with a Maxwellian law for the true distribution function of rotational velocities among $B$ and $\mathrm{A}$ stars in the main-sequence band (Deutsch, 1967). The Maxwellian law was found to be admissable for stars in the range B5-B9 and for those in the range A3 to F0. However, in the ranges B2-B5 and B9-A2 the incidence of sharp-line stars appeared to be excessively high, as Conti (1965) had first pointed out for stars near A0V. In these parts of the main-sequence band, the line-width statistics were found to be compatible with bimodal Maxwellian distributions. The conjecture was put forward that the excessively abundant sharp-line stars represent older populations, which consist of metamorphs of red giants, or Population I analogues of 'horizontal-branch stars'. However, no reason was given as to why the line-widths statistics failed to show similar excesses of sharp-line stars in the ranges of spectral types B5-B9 and A2-F0.

The peculiar A stars and the metallic line stars introduce serious ambiguities into the spectral classification of A-type stars. According to the photometry of Eggen (1963) and others, one can best circumvent this difficulty by grouping nearby A stars on a criterion of color-class. Accordingly, this was done for the A stars in the 1965 work. However, it was not done for the B stars. This resulted in the exclusion from the statistics of a number of Ap stars with the colors of B stars.

\section{Simple Maxwellian Distributions}

At the same time that we correct this inconsistency in the 1965 work, we are able to incorporate additional measurements of $V \sin i$ that have recently been made on the system of Slettebak and Howard. In advance of publication, Dr. G. W. Preston has kindly made available to me his determinations of $V \sin i$ for all known Ap stars 
brighter than $V=6.0$ and north of $-15^{\circ}$ declination. These comprise 18 objects with (unreddened) colors in the range $-0.05 \leqslant(B-V)_{0} \leqslant \pm 0.10$, and 43 objects in the range $-0.18 \leqslant(B-V)_{0} \leqslant-0.03$.

In these ranges of $(B-V)_{0}$, which for normal stars correspond to spectral-type ranges $\mathrm{B} 5$ - to $\mathrm{B} 9+$ and $\mathrm{B} 9+$ to $\mathrm{A} 2+$, respectively, the line-widths of Slettebak are complete only to $V=5.0$. To combine the two sets of data, therefore, I have extrapolated the Slettebak measurements to $V=6.0$ by applying the factor 4 to the numbers of stars he found in each increment of $V \sin i$. The parentheses in Table I and in the figures are intended to denote that the corresponding numbers have been obtained by such an extrapolation. The same extrapolation has been made to obtain the 'observed' numbers of stars given in the respective boxes of the histograms.

In the range of spectral types B2-B5, inclusive, the measurements of Walker and Hodge (1966) give the line-widths $V \sin i$ for many more stars than were measured by Slettebak and Howard (1955). The bright limit of the main-sequence band was arbitrarily set at 1.5 magnitudes above the zero-age main sequence, and the linewidths were counted for all the stars that Walker and Hodge assigned to this strip in the Hertzsprung-Russell diagram.

Table I shows the numbers of stars for which $V \sin i$ was taken to be known,

TABLE I

\begin{tabular}{lccc}
\hline \multicolumn{4}{c}{ Maxwellian distributions for $V \sin i$} \\
\hline $\begin{array}{l}\text { Sp. Type } \\
(B-V)_{0}\end{array}$ & B2 to B5 & B5 - to B9 + & B9 + to A2 + \\
$N$ (normal) & -0.24 to -0.16 & -0.18 to -0.03 & -0.05 to +0.10 \\
$N($ pec.) & 197 & $(208)$ & $(316)$ \\
$1 / j(\mathrm{~km} / \mathrm{sec})$ & 185 & 43 & 18 \\
$V \sin i<50$ & 185 & 164 & 151 \\
& 81 & 53 & 90 \\
Obsd. & 81 & 23 & 14 \\
Pred. & 13 & & \\
\hline
\end{tabular}

according to these precepts, in each of three intervals along the main-sequence band. In the interval B2-B5, of course, there is no subgroup of stars that are recognizable analogues of the Ap stars encountered between B5 and A2.

For the stars counted in each range of spectral type, Table I also gives the rootmean-square value of $V \sin i$. This is the parameter $1 / j$; it is equal to the modal value of the true rotational velocities, if these have a Maxwellian distribution. With these values of $1 / j$, and the formulae given in Deutsch (1967), one can then predict, for each sample of $N$ stars, how many should have $V \sin i<50 \mathrm{~km} / \mathrm{sec}$. Evidently these predictions are grossly incorrect; each of the three samples of Table I contains many more sharp-line stars than would be found if the true distribution were Maxwellian. In particular, on including the Ap stars with color-classes in the range B5 - to B9+, we now find an abnormality in the statistics where none appeared in the 1967 work. The present position is that all along the main-sequence band from B2 to A2, slow 
rotators have far too large an incidence to comport with a Maxwellian law for the true distribution of rotational velocities.

This result is likely to be of greater significance than one might think. For, by using the methods of classical statistical mechanics, one can rigorously prove the following theorem relative to the angular velocities $\omega$ of the stars. If the distribution of $\boldsymbol{\omega}$ is isotropic, and if the component $\omega_{x}$ in a system of Cartesian coordinates is distributed independently of $\omega_{y}$ and $\omega_{z}$, then the distribution of the positive scalar $\omega$ is given by $f(j \omega) d(j \omega)$, where $f$ is the Maxwellian law,

$$
f(x)={ }_{\sqrt{ } \pi}^{4} x^{2} \exp \left(-x^{2}\right) ;
$$

or by the sum $\sum_{i} a_{i} f\left(j_{i} \omega\right) d\left(j_{i} \omega\right)$, with, $\sum_{i} a_{i}=1$. The proof of this theorem is given in Appendix I.

Now, the angular-velocity vectors of stars are known to be directed at random, and one would suppose that little or no coupling should occur among $\left(\omega_{x}, \omega_{y}, \omega_{z}\right)$ for objects that rotate about one of their principal axes of inertia. Therefore our theorem ought to apply. However, the results in Table I have ruled out simple Maxwellian distributions for stellar rotations. Accordingly, we shall now try to represent the observed statistics with curves derived from bimodal Maxwellian laws, for these represent the next most simple distributions which our theorem will allow.

\section{Bimodal Maxwellian Distributions}

To obtain the parameters for the bimodal distributions, recourse was had to a computer program written by Mr. J. F. Bartlett, of the Booth Computing Center at the California Institute of Technology. Bartlett's program finds the best-fitting bimodal distributions on the basis of a maximum-likelihood calculation, starting from the histograms that have been observed. The smooth curves in the figures give the distributions of $V \sin i$ that would be produced by these optimum bimodal Maxwellian laws for $V$. Each curve is the weighted sum of two apparent distributions; in units of percent per $\mathrm{km} / \mathrm{sec}$, these apparent distributions are, respectively,

where

$$
\begin{aligned}
\varphi_{y} & =100 j_{y} \varphi\left(j_{y} V \sin i\right) \\
\varphi_{0} & =100 j_{0} \varphi\left(j_{0} V \sin i\right),
\end{aligned}
$$

$$
\varphi(x)=2 x \exp \left(-x^{2}\right)
$$

The numerical parameters given in Figure 1, then, have the following significance: $53 \%$ of the 197 stars represented belong to a 'young' population having a Maxwellian distribution of $V$ with a mode $1 / j_{y}=248 \mathrm{~km} / \mathrm{sec}$; and $47 \%$ belong to an 'old' population having a Maxwellian distribution of $V$ with a mode $1 / j_{0}=39 \mathrm{~km} / \mathrm{sec}$. Because no account was taken of the distribution of $V \sin i$ within the first box $(V \sin i<50 \mathrm{~km} / \mathrm{sec})$, it is necessary to add that these calculations really determine only an upper limit to the parameter $1 / j_{0}$, except possibly in the range B2-B5. 


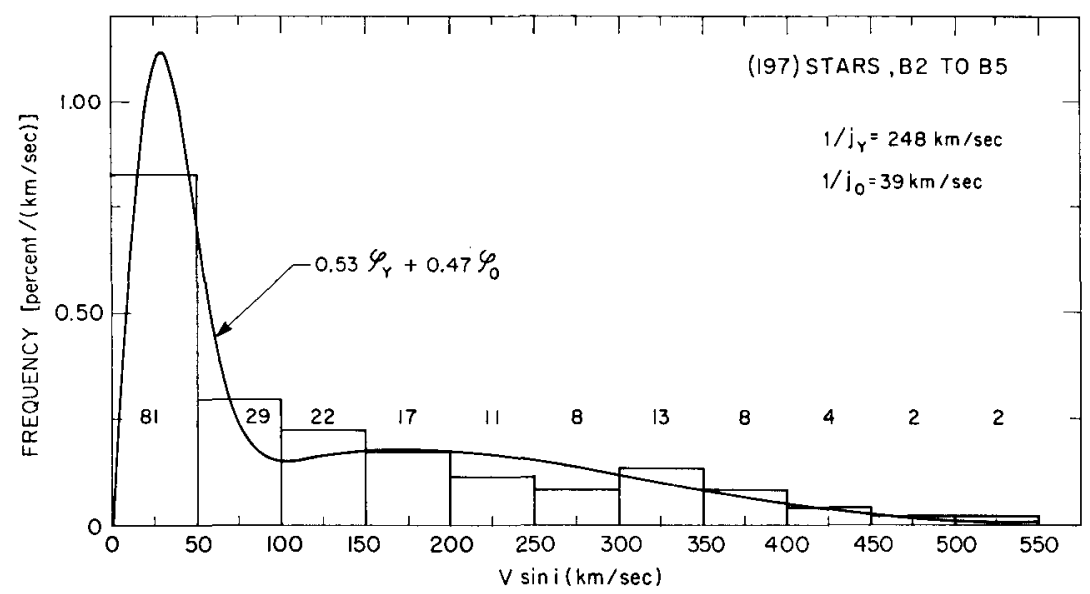

Fig. 1. Distribution of rotational velocities of early $B$ stars in the main-sequence band, according to the measures of Walker and Hodge (1966). The smooth curve is derived from a bimodal Maxwellian distribution for the true rotational velocities $V$.

\section{Interpretation of the Statistics}

The chi-square test indicates that one cannot reject these bimodal Maxwellian distributions on statistical grounds. However, one need not accept the hypothesis that the 'extra' slow rotators comprising the $\mathrm{O}$ Population are really metamorphs of red giants. Indeed, a number of objections can now be lodged against this suggestion. The chief difficulty concerns the kinematics. If the stars of the O Population originally lay on the main-sequence as late $\mathrm{A}$ or $\mathrm{F}$ stars, their motions should still be distinctively larger than the Y Population stars which they now resemble. For example, in the compilation of Allen (1963), the mean velocity dispersion is $20 \mathrm{~km} / \mathrm{sec}$ at A0, $29 \mathrm{~km} / \mathrm{sec}$ at F0, and $42 \mathrm{~km} / \mathrm{sec}$ at G0. The possibility exists that the O Population stars near A0 have somewhat brighter absolute magnitudes than the Y Population stars. Since this has not been taken into account in the calculation of their motions, the latter may have been systematically underestimated. However, after a review of the absolute-magnitude and velocity determinations for the stars near A0V (Eggen, 1963), it seems most unlikely that the O Population stars can have a velocity dispersion which is much larger than that of the Y Population stars.

Other objects that have been interpreted as possible metamorphs of red giants are the blue stragglers that are found in M 67 and several other old open clusters. The A stars in M 67 have a mean rotational velocity of nearly $100 \mathrm{~km} / \mathrm{sec}$ (Deutsch, 1966) - a value which is awkwardly intermediate between the mean rotational velocities of the two populations that seem discernible among the early A stars in the field (Figure 3). Spectroscopic determinations of $g$ and $M$ have been reported by Sargent (1968) for three of the early A stars in M 67. His result for the mean mass is $0.7 \pm 0.2 m_{\odot}$. This is low for ordinary main-sequence A stars, leading Sargent to conclude " $\ldots$ that these three stars do constitute the blue end of a thinly populated horizontal branch in M 67". 
However, Sargent (1969) now agrees that this mass-determination is sufficiently sensitive to observational errors and to uncertainties in the broadening theory for the Balmer lines, that it cannot really rule out the larger masses corresponding to ordinary main-sequence A stars.

Rodgers (1968) has reported a similar mass-determination for the field star HD 6870. The motion of this object is such that it could be a horizontal-branch star belonging to the old moving group associated with $\sigma$ Puppis (Eggen, 1964). Rodgers concludes that HD 6870 probably is a member of this moving group. However, he also concludes that its mass is nearly normal for the spectral type of a main-sequence star, and that the object is therefore probably not a horizontal-branch star.

Meanwhile, Deutsch (1969) has obtained a similar result for Fagerholm 190, an A8 star which is one of the blue stragglers in M 67. In this instance, no mass-determination has been made. However, the star appears to be a single-line spectroscopic

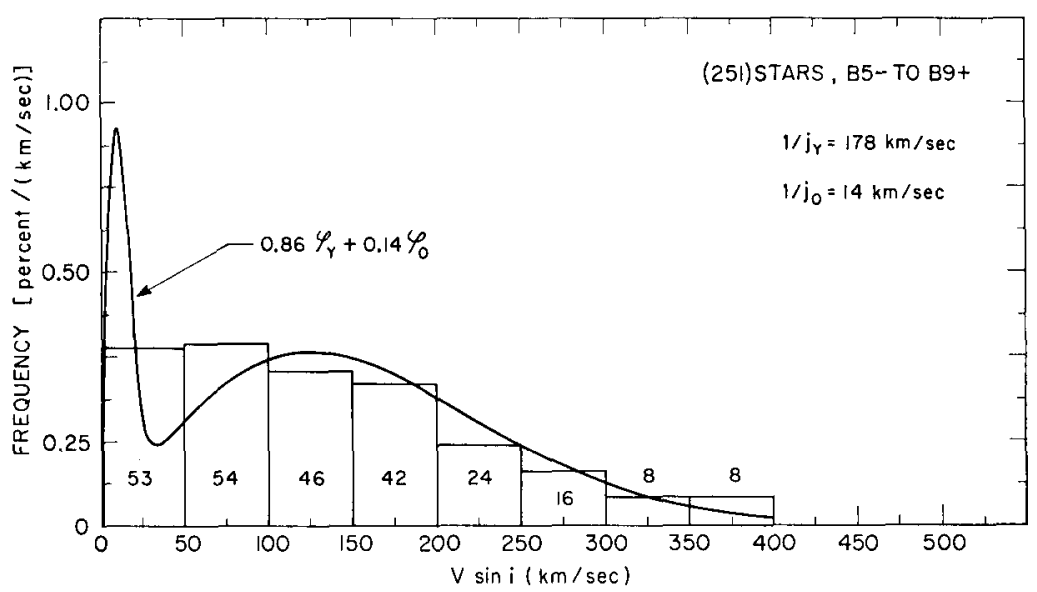

Fig. 2. Distribution of rotational velocities of late B stars, in the main-sequence band, according to the measures of Slettebak (1954). The statistics include Preston's (1969a) measures for 43 Ap stars that have the color-classes of late B stars. The smooth curve is derived from a bimodal Maxwellian law for $\boldsymbol{V}$.

binary, with a period of four days and a low mass-function. The object therefore cannot be a metamorph of a red giant. Most probably it has reached its present position in the color-magnitude diagram by the accretion of mass from its companion, according to the McCrea-Kippenhahn theory of evolution in close binary systems.

Van den Heuvel (1968) has shown that after the transfer of mass from the original primary of a close pair, the new primary is likely to have a rotational velocity appreciably lower than most stars of the same mass, which have not experienced accretion from an evolving companion. Moreover, he has recently (1969) concluded that " ... at least $11.7 \%$ (and possibly up to $22 \%$ ) of the main-sequence stars earlier than A5 are expected to be CBR" - i.e., close-binary remnants of a McCrea-Kippenhahn evolutionary process. The work of Van den Heuvel also shows that the incidence of CBR 
may go appreciably higher at some spectral types, depending on the incidence of close binaries and on the fraction of mass that is lost to the system when the original primary overflows its Roche lobe.

On the basis of these arguments, it therefore seems plausible to regard most of the 'extra' slow rotators of our O Population as CBR. However, we still lack a reliable

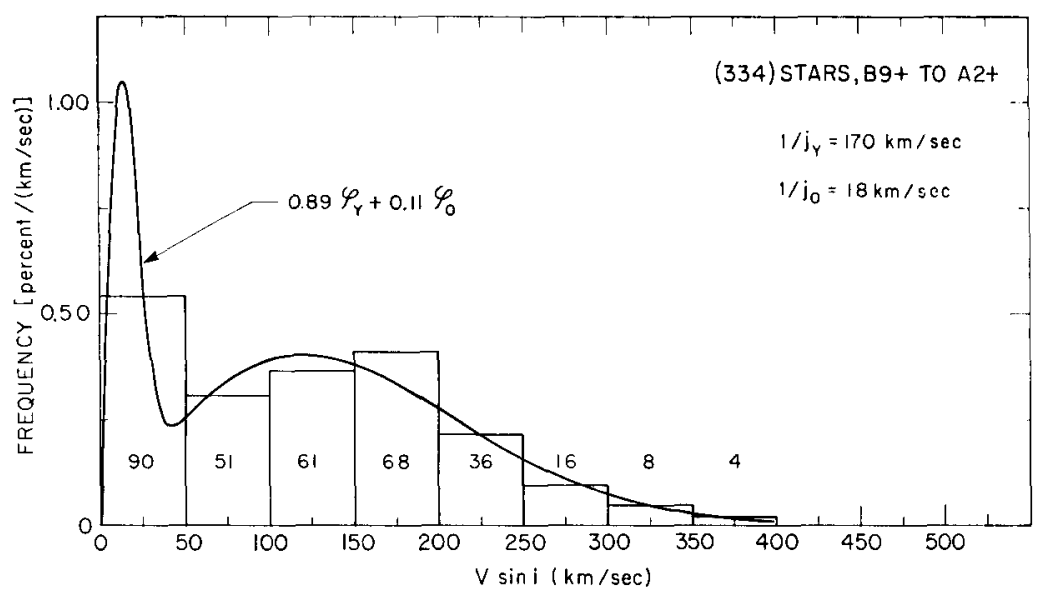

Fig. 3. Distribution of rotational velocities of early $\mathrm{A}$ stars in the main-sequence band, according to the measures of Slettebak (1955). The statistics include Preston's (1969a) measures for 18 Ap stars that have the color-classes of early A stars. The smooth curve is derived from a bimodal Maxwellian law for $V$.

spectroscopic discriminant between those sharp-line stars that are CBR and those that are not. Thus, of the 53 stars counted in the first box of Figure 2, 29 are known to be Ap stars. Ten more Ap stars lie in the second box, and two each in the next two boxes. From the parameters for the best-fitting bimodal distribution, one ought to find 17 of the Y Population stars in the first box, and 35 of the O Population. Similarly one expects that in the first box of Figure 3 there ought to be 22 Y Population stars and $68 \mathrm{O}$ Population stars. The box actually contains $14 \mathrm{Ap}$ stars; three more lie in the second box and one in the third. These results hardly permit one to assign the Ap stars to one population or the other.

It remains to note that not all the 'extra' slow rotators we have found in the field need be CBR. Indeed, Preston (1969b) has shown that some of the Ap stars appear to have been subject to a different and very powerful kind of rotational deceleration, which may arise from an interaction between the stellar magnetic fields and the interstellar medium. Mestel (1969) has recently described the torques on a star that support a stellar wind and a dipole field inclined to the rotation axis. Other modes of rotationai braking have been briefly reviewed by Huang and Struve (1960).

If the creation function for early-type stars is constant with time, and Maxwellian with parameter $1 / j_{0}$; and if all stellar rotations are subject to a similar, exponential decay; then the steady-state distribution function for rotational velocities can be 
shown to be $G_{T}\left(j_{0} V\right) d\left(j_{0} V\right)$,

where

$$
G_{T}(x)=\frac{4 T}{\sqrt{ } \pi x} \int_{x}^{x e^{1 / T}} z^{2} e^{-z^{2}} d z .
$$

In this expression, $T$ is the decay time for stellar rotations, expressed in units of the lifetime in the main-sequence band. The function $G_{T}(x)$ is actually a limiting case of a sum of Maxwellian distributions having all values of $1 / j$ between $1 / j_{0}$ and zero (see Appendix II). The corresponding distribution of $V \sin i$ is $J_{T}\left(j_{0} V \sin i\right) d\left(j_{0} V \sin i\right)$, where

$$
J_{T}(x)=\int_{0}^{\pi / 2} G_{T}(x / \sin i) d i
$$

The function $G_{T}(x)$ is a special case of the distribution functions for rotationallybraked stars, that have been discussed by Huang (1967). The corresponding function $J_{T}(x)$ is characterized by the two free parameters $1 / j_{0}$ and $T$.

One finds that $J_{T}(x)$ can represent the statistics of $V \sin i$ better than can the function $\varphi(x)$ which is derived from the simple Maxwellian law, and which has one free parameter. However, $J_{T}(x)$ is less successful than the distribution derived from a bimodal Maxwellian law, which has three parameters. Little attention has been paid in this paper to the use of $J_{T}(x)$, for the work of Van den Heuvel indicates that the McCrea-Kippenhahn evolution of close binary pairs is likely to have produced most or all of the 'extra' slow rotators that lie on the early main-sequence.

\section{Appendix I. The Distribution Function for the Magnitude of a Vector that has Random Orientation}

It is required to find the distribution function of a positive scalar $\omega$, which is the magnitude of a vector $\omega$. We assume that the distribution of $\omega$ is isotropic. We also assume that if $\omega$ is decomposed into components along Cartesian axes, the distribution of any component (say, $\left.\omega_{x}\right)$ is independent of the other components $\left(\omega_{y}\right.$ and $\omega_{z}$ in this case).

Let us designate $\Omega$ as the non-dimensional quantity $j \omega$, where $j$ is a parameter with the dimensions of $\omega^{-1}$, and where

$$
\boldsymbol{\Omega}=\mathrm{i} \Omega_{x}+\mathrm{j} \Omega_{y}+\mathrm{k} \Omega_{z} .
$$

The probability that $\Omega_{x}$ lies in $d \Omega_{x}, \Omega_{y}$ in $d \Omega_{y}$, and $\Omega_{z}$ in $d \Omega_{z}$ is then

$$
F\left(\Omega_{x}, \Omega_{y}, \Omega_{z}\right) d \Omega_{x} d \Omega_{y} d \Omega_{z}=h\left(\Omega_{x}^{2}\right) h\left(\Omega_{y}^{2}\right) h\left(\Omega_{z}^{2}\right) d \Omega_{x} d \Omega_{y} d \Omega_{z} .
$$

The assumption of isotropy also insures that we may write

$$
F\left(\Omega_{x}, \Omega_{y}, \Omega_{z}\right)=H\left(\Omega^{2}\right)=H\left(\Omega_{x}^{2}+\Omega_{y}^{2}+\Omega_{z}^{2}\right) .
$$


If

then

$$
\Omega_{x}=\Omega_{y}=0, \quad \text { and } \quad \Omega_{z}=\Omega
$$

$$
H\left(\Omega^{2}\right)=h^{2}(0) h\left(\Omega^{2}\right) ;
$$

and therefore by Equation (2)

$$
h\left(\Omega_{x}^{2}\right) h\left(\Omega_{y}^{2}\right) h\left(\Omega_{z}^{2}\right)=h^{2}(0) h\left(\Omega^{2}\right) .
$$

Now for any $u \geqslant 0$, let

$$
\xi(u)=\ln \frac{h(u)}{h(0)} .
$$

Then by Equation (5)

$$
\begin{aligned}
\xi\left(\Omega^{2}\right) & =\ln \frac{h\left(\Omega_{x}^{2}\right)}{h(0)}+\ln \frac{h\left(\Omega_{z}^{2}\right)}{h(0)}+\ln \frac{h\left(\Omega_{z}^{2}\right)}{h(0)} \\
& =\xi\left(\Omega_{x}^{2}\right)+\xi\left(\Omega_{y}^{2}\right)+\xi\left(\Omega_{z}^{2}\right) .
\end{aligned}
$$

In particular, if $\Omega_{x}^{2}=\Omega_{y}^{2}=u$, and $\Omega_{z}=0$,

$$
\xi(2 u)=2 \xi(u) \text {. }
$$

Again, if $\Omega_{x}^{2}=u, \Omega_{y}^{2}=2 u$, and $\Omega_{z}=0$,

$$
\xi(3 u)=\xi(u)+\xi(2 u)=3 \xi(u) ;
$$

and, by induction, for any positive integer $n$,

If we let

$$
\xi(n u)=n \xi(u)
$$

then

$$
u=v / n
$$

whence

$$
\xi[n(v / n)]=n \xi(v / n)
$$

$$
\xi(v / n)=\frac{1}{n} \xi(v) .
$$

If $m$ is another positive integer, then Equation (13) yields

$$
m \xi(v / n)=\frac{m}{n} \xi(v)
$$

and by Equation (10),

$$
\xi\left(\frac{m}{n} v\right)=\frac{m}{n} \xi(v) .
$$

Since this result is valid for any positive rational number $(m / n)$, by continuity it will 
also be valid for any positive irrational number $x$; viz.,

$$
\xi(x v)=x \xi(v) .
$$

in particular, if $v=1$,

Therefore

$$
\xi(x)=x \xi(1)=c x .
$$

$$
d \xi(x) / d x=c,
$$

and, by Equation (6),

$$
\frac{1}{h(x)} \frac{d h}{d x}=c,
$$

the solution of which is

$$
h(x)=a e^{c x} .
$$

Since $h\left(\Omega_{x}^{2}\right)$ is a probability function, the appropriate normalization condition is

$$
1=2 \int_{0}^{\infty} h\left(\Omega_{x}^{2}\right) d \Omega_{x}=2 a \int_{0}^{\infty} e^{c \Omega_{x^{2}}} d \Omega_{x},
$$

and the mean value of $\Omega_{x}^{2}$ is

$$
\left\langle\Omega_{x}^{2}\right\rangle=2 a \int_{0}^{\infty} \Omega_{x}^{2} e^{c \Omega_{x^{2}}} d \Omega_{x} .
$$

If we define the parameter $j$ in the relation

$$
(1 / j)^{2}=2\left\langle\omega_{x}^{2}\right\rangle,
$$

we then find that Equations (21) and (22) yield the results $a=\sqrt{ } \pi$, and $c=-1$.

We may now write the distribution of $\Omega$ in the form $f(\Omega) \mathrm{d} \Omega$, where

$$
\begin{aligned}
f(\Omega) & =\iint_{\Omega=\text { const }} H\left(\Omega^{2}\right) \Omega^{2} \sin \vartheta d \vartheta d \varphi \\
& =4 \pi \Omega^{2} H\left(\Omega^{2}\right) .
\end{aligned}
$$

Adducing Equation (4), we find that

$$
f(\Omega)=4 \pi \Omega^{2} h^{2}(0) h\left(\Omega^{2}\right),
$$

and, from Equation (20),

$$
\begin{aligned}
f(\Omega) & =4 \pi \Omega^{2} a^{2} e^{c \Omega^{2}} \\
& =\frac{4}{\sqrt{ } \pi} \Omega^{2} e^{-\Omega^{2}} .
\end{aligned}
$$

This is the Maxwellian distribution. 
Now suppose that the distribution is bimodal, with parameters $j_{1}$ and $j_{2}$, respectively. Let $a_{1}$ be the incidence of one distribution and $a_{2}=1-a_{1}$ the incidence of the other. Then the probability that $\boldsymbol{\omega}$ lies in $d \omega_{1}, d \omega_{2}, d \omega_{3}$ may be written as

$$
a_{1} F\left(\Omega_{x}, \Omega_{y}, \Omega_{z}\right) d \Omega_{x} d \Omega_{y} d \Omega_{z}+a_{2} F\left(\Omega_{x}^{\prime}, \Omega_{y}^{\prime}, \Omega_{z}^{\prime}\right) d \Omega_{x}^{\prime} d \Omega_{y}^{\prime} d \Omega_{z}^{\prime},
$$

where $\Omega_{x}=j_{1} \omega_{x}, \Omega_{x}^{\prime}=j_{2} \omega_{x}$, etc., etc. The derivation of each distribution then proceeds as before, and we obtain for the composite distribution the bimodal Maxwellian law,

$$
f\left(\Omega_{1}\right)+f\left(\Omega_{2}\right)=\frac{4}{\sqrt{ } \pi} a_{1} \Omega_{1}^{2} e^{-\Omega_{1}^{2}}+\frac{4}{\sqrt{ } \pi} a_{2} \Omega_{2}^{2} e^{-\Omega_{2}^{2}} .
$$

The probability that $\omega$ lies in $d \omega$ is then

$$
f\left(j_{1} \omega\right) d\left(j_{1} \omega\right)+f\left(j_{2} \omega\right) d\left(j_{2} \omega\right) .
$$

\section{Appendix II. The Steady-State Distribution Function Corresponding to Decelerating Maxwellian Distributions}

Suppose that in unit volume near the sun, the rate of creation of stars with (nondimensional) angular velocities $j_{0} \omega_{0}$ in $d\left(j_{0} \omega_{0}\right)$ is $N f\left(j_{0} \omega_{0}\right) d\left(j_{0} \omega_{0}\right)$, where $N$ is a constant; $f$ is the Maxwellian law,

and

$$
f\left(j_{0} \omega_{0}\right)=\frac{4}{\sqrt{ } \pi}\left(j_{0} \omega_{0}\right)^{2} \mathrm{e}^{-\left(j_{0} \omega_{0}\right)^{2}}
$$

$$
1 / j_{0}=\left(\frac{2}{3}\right)^{1 / 2}\left\langle\omega_{0}^{2}\right\rangle^{1 / 2}=\left\langle\left(\omega_{0} \sin i\right)^{2}\right\rangle^{1 / 2} .
$$

Suppose, further, that the angular velocity of every star decays according to the law

$$
\omega=\omega_{0} e^{-\tau / T} .
$$

In this expression, $\tau$ is the age of the star, and $T$ the decay-time for the angular velocity. We take the unit of time to be the whole main-sequence lifetime of the star, so that $0<\tau<1$.

For the incidence of stars with angular velocities in $d\left(j_{0} \omega\right)$ and ages in $d \tau$, we then have

where

$$
\begin{aligned}
g_{T}\left[j_{0} \omega\right] d\left[j_{0} \omega\right] d \tau & =f\left[\left(j_{0} \omega\right) e^{\tau / T}\right] d\left[\left(j_{0} \omega\right) e^{\tau / T}\right] d \tau \\
& =f(j \omega) d(j \omega) d \tau
\end{aligned}
$$

$$
1 / j=\left(1 / j_{0}\right) e^{-\tau / T} .
$$

Therefore, among stars created at a given epoch, the distribution of $j \omega$ remains Maxwellian, but the parameter $1 / j$ decays according to Equation (5).

In a steady state, stars of all ages $0<\tau<1$ will contribute to the incidence in any 
increment $d\left(j_{0} \omega\right)$. We may write this distribution as $G_{T}\left(j_{0} \omega\right) d\left(j_{0} \omega\right)$, where

$$
\begin{aligned}
G_{T}(x) & =\int_{0}^{1} g(x) d \tau \\
& =T \int_{0}^{1 / T} e^{\tau / T} f\left(x e^{\tau / T}\right) d(\tau / T),
\end{aligned}
$$

which may be reduced to

$$
G_{T}(x)=\frac{4 T}{\sqrt{\pi x}} \int_{x}^{x e^{1 / \tau}} z^{2} e^{-z^{2}} d z .
$$

The corresponding distribution of $V \sin i$ is

where

$$
J_{T}\left(j_{0} V \sin i\right) d\left(j_{0} V \sin i\right),
$$

$$
J_{T}(x)=\int_{0}^{\pi / 2} G_{T}(x / \sin i) d i
$$

\section{Acknowledgements}

I gratefully acknowledge numerous helpful discussions with G. W. Preston concerning the subject of this paper. My thanks also go to D. M. Peterson who improved my proof of the theorem given in Appendix I; and to J. F. Bartlett, who obtained the bimodal Maxwellian distributions drawn in the figures.

\section{References}

Allen, C. W.: 1963, Astrophysical Quantities, 2nd ed., Athlone Press, London, p. 243.

Conti, P. S.: 1965, Astrophys. J. 142, 1594.

Deutsch, A. J.: 1966, Astron. J. 71, 383.

Deutsch, A. J.: 1967, The Magnetic and Related Stars, (ed. by R. C. Cameron), Mono Book Corp., Baltimore, p. 181.

Deutsch, A. J.: 1969, reported by H. W. Babcock in C.I.W. Yrbk. 68.

Eggen, O. J.: 1963, Astron. J. 68, 697.

Eggen, O. J.: 1964, Astron. J. 69, 570.

Huang, S.-S.: 1967, Astrophys. J. 150, 229.

Huang, S.-S. and Struve, O.: 1960, in Stellar Atmospheres, (ed. by J. L. Greenstein), Univ. of Chicago Press, p. 321.

Mestel, L.: 1969, Monthly Notices Roy. Astron. Soc. 140, 177.

Preston, G. W.: 1969a, private communication.

Preston, G. W.: 1969b, this volume, p. 254.

Rodgers, A. W.: 1968, Astrophys. J. 152, 109.

Sargent, W. L. W.: 1968, Astrophys. J. 152, 885.

Sargent, W. L. W.: 1969, private communication. 
Slettebak, A.: 1954, Astrophys. J. 119, 146.

Slettebak, A.: 1955, Astrophys. J. 121, 653.

Slettebak, A. and Howard, R. F.: 1955, Astrophys. J. 121, 102.

Van den Heuvel, E. P. J.: 1968, Bull. Astron. Inst. Neth. 19, 326.

Van den Heuvel, E. P. J.: 1969, Lick Obs. Bull., No. 603.

Walker, G. A. H. and Hodge, S. M.: 1966, Pub. Dominion Astrophys. Obs. 13, 2.

\section{Discussion}

Van den Heuvel: I want to make a comment on what Dr. Deutsch just said. When I said yesterday that the existence of the slow rotators of type $\mathbf{B} 8$ and later could perhaps be explained by the closebinary evolution process, I did not imply that the same would not work at earlier spectral types. However, my material presented yesterday did not allow such a conclusion. I already suggested some years ago (Van den Heuvel, 1967, 1968a), however, that the close-binary evolution is the explanation for the occurrence of slow rotators (Van den Heuvel, 1966, 1968b) among the B as well as the A stars. Van den Heuvel, E. P. J.: 1966, Proc. Koninkl. Nederl. Akademie van Wetenschappen, Series B, 69, 357. Van den Heuvel, E. P. J.: 1967, Observatory 87, 68.

Van den Heuvel, E. P. J.: 1968a, Bull. Astron. Inst. Neth. 19, 326.

Van den Heuvel, E. P. J.: 1968b, Bull Astron. Inst. Neth. 19, 309.

$A b t$ : By grouping the Ap stars with the rapid rotators, are you implying that Ap stars are rapid rotators seen pole-on?

Deutsch: No. The hypothesis was that nearly all the slow rotators in the Y population will show the Ap characteristic, regardless of aspect. Of course, most will be seen at high inclination.

Kraft: The excess of sharp-lined B8-A2 stars is also shown in young clusters such as $\alpha$ Per and the Pleiades. This suggests that the two populations are not an 'old' vs. 'young' effect, but rather that the sharp-lined group results from a selective continuous decay of rotation in some stars as the stars age.

Deutsch: I agree.

Hardorp: The resolution in $V \sin i$ is not constant along the $V \sin i$-axis. It therefore is justified to use narrower boxes for the slow rotations. If you make the first box $25 \mathrm{~km} / \mathrm{sec}$ instead of 50 , you find the over-abundance of slow rotators also in Slettebak's old samples of B6-B9 stars as well as in the B2-B5 sample.

Deutsch: I agree. 